\title{
La implementación del Plan Estratégico Nacional de Mercados Verdes en el Departamento de Amazonas, Colombia, 2002- 2011: ¿Acierto o desacierto?
}

\author{
Implementation of the National Strategic Plan for Green Markets in the Province of Amazonas, Colombia, \\ 2002-2011: Rightness or Wrongness \\ Luz Marina Mantilla Cárdenas ${ }^{(1)}$ \\ (1) Magister en Gobierno y Políticas Públicas, Universidad Externado de Colombia, Columbia University. Directora General, Instituto Ama- \\ zónico de Investigaciones Científicas - SINCHI. luzmarmantilla@sinchi.org.co
}

Recibido 21 de julio de 2015. Modificado 23 de julio de 2015. Aprobado 27 de julio de 2015.

DOI: http://dx.doi.org/10.16924/riua.v0i42.844

\begin{abstract}
Palabras clave
Gobernanza, bienes comunes, articulación, implementación, políticas públicas.

\section{Resumen}

En 2002, el Ministerio de Ambiente presentó el Plan Estratégico Nacional de Mercados Verdes - PENMV, con el objetivo de consolidar la producción de bienes y servicios ambientales y aprovechar la oferta de la biodiversidad colombiana. Este plan no fue implementado de forma adecuada en el departamento de Amazonas, dado que carece de un modelo de gestión que defina la articulación de la entidad territorial con las instituciones encargadas del tema y la participación ciudadana. De hecho, al analizar los planes de desarrollo nacionales y los de las gobernaciones de dicho departamento, no se menciona este plan. Se presentan las iniciativas más importantes de manejo sostenible de la biodiversidad, teniendo en cuenta el PENMV, desarrolladas por personas de la región en conjunto con instituciones como el Instituto SINCHI y Corpoamazonia.
\end{abstract}

\section{Key words}

Government, common goods, articulation, implementation, public politics

\begin{abstract}
In 2002, the Colombian Ministry of the Environment elaborated the National Strategic Plan for Green Markets to consolidate the production of environmental goods and services and take advantage of what the country's biodiversity has to offer. The PENMV was not implemented accurately in the province of Amazonas, due to the inexistence of a model to define how the province, the institutions involved in the topic and the citizen participation should be articulated. When a review of the governmental plans implemented at different points in time in the Amazon province is undertaken, no informationation can be found; thus evidencing the inexistence of that articulation. What it is presented are the main initiatives in the sustainable management of biodiversity developed by some local actors under actions implemented by national institutions such as the SINCHI Institute and Corpoamazonia that are supported in the framework of the plans.
\end{abstract}

\section{INTRODUCCIÓN}

En 2002, el Ministerio del Medio Ambiente presentó el Plan Estratégico Nacional de Mercados Verdes, en adelante -PENMV—, con el objetivo de consolidar la producción de bienes ambientalmente sostenibles e incrementar la oferta de servicios ecológicos competitivos en los mercados nacionales e internacionales contribuyendo a mejorar la calidad ambiental y el bienestar social. Adicionalmente, clasificó los mercados verdes en cuatro categorías: i) mecanismo de desarrollo limpio, ii) aprovechamiento sostenible de recursos naturales que incluye: productos naturales no maderables, agricultura ecológica, biotecnología y productos naturales maderables; iii) ecoproductos industriales; y iv) servicios ambientales (Ministerio de Ambiente, 2002).

En la primera parte de este artículo se tratan los conceptos clave de política pública, gobernanza y medio ambiente. Luego, a partir de la premisa de que el PENMV no fue implementado adecuadamente en el departamento de Amazonas se analizará el modelo de gestión del PENMV en dicho departamento y 
su articulación con los Planes de Desarrollo departamentales entre 2002 y 2011. Se estudiará el modelo de gestión nacional que materializa los objetivos del PENMV y se presentarán experiencias departamentales derivadas de los mercados verdes, surgidas gracias a la participación de entidades como el Instituto SINCHI y Corpoamazonia, para concluir que la implementación del PENMV no fue adecuada, porque no existe un modelo de gestión que defina la articulación de la entidad territorial, con las instituciones nacionales competentes en el tema y los actores involucrados.

\section{LA IMPLEMENTACIÓN DE LA POLÍTICA PÚBLICA Y LOS Mercados Verdes}

En la década de los años setenta se demostró que muchas políticas y programas sociales no habían sido tan efectivos como se creía, dado que no resolvieron los problemas y su impacto no fue evidente. De ahí que los estudiosos de las políticas públicas enfocaran su atención en los insumos internos, los resultados y los impactos. Este énfasis en los aspectos de la implementación y los resultados de las políticas públicas marcaron una revolución en el discurso de la gobernanza (Parson, 2007).

Los procesos de adopción e implementación de políticas públicas son tan importantes como la formulación, lo cual debe ser entendido por los responsables de la formulación de las políticas públicas (Stain, 2006). El enfoque Top down es el que más se aproxima al análisis de la construcción del PENMV. La práctica muestra que la implementación es un proceso de interacción entre el establecimiento de metas y las acciones emprendidas para alcanzarlas. Las metas deben estar claramente definidas y entenderse; los recursos deben estar disponibles; la secuencia de órdenes debe ser capaz de ensamblar y controlar los recursos; y el sistema debe ser capaz de comunicar efectivamente y controlar a los individuos y a las organizaciones que participan en el desempeño de las tareas.

La importancia de las políticas públicas y su implementación para el manejo sostenible de la biodiversidad requieren de la interacción con los actores directos. La Evaluación de Ecosistemas del Milenio (WRI 2005) menciona que un conjunto efectivo de respuestas que aseguren una gestión sostenible de los ecosistemas, requiere cambios sustanciales en las instituciones y en la gobernanza, en las políticas económicas e incentivos, en los factores sociales y de comportamiento y en la tecnología y los conocimientos, los cuales podrían disminuir de manera considerable la severidad de estos problemas en las próximas décadas.

La forma en que las personas se organizan para tomar decisiones, ejecutar actividades, definir los procedimientos y normas que regulan sus relaciones, acuerdos y transacciones, se denomina gobernanza. Así, la articulación productiva se debe entender como "la construcción de ventajas competitivas que surgen de las interrelaciones creadas y desarrolladas entre empresas e instituciones; es uno de los instrumentos y herramientas que los gobiernos utilizan para crear condiciones y ambientes técnico institucionales y económicos donde estas interrelaciones tengan lugar de forma beneficiosa para la sociedad” (Barriga et al., 2007).

El modelo de gestión para el manejo sostenible de la biodiversidad se define, en este caso, como un marco de referencia para la administración de los emprendimientos que se incorporan a la cadena de valor y que requieren una participación horizontal de los actores involucrados, en pro de gestionar los bienes comunes ${ }^{1}$ a los que están asociadas las comunidades.

\section{CARACTERIZACIÓN PRODUCTIVA DEL DEPARTAMENTo de Amazonas}

La estructura productiva del departamento de Amazonas está en: producción agrícola; colecta y extracción de productos del bosque y, en la pesca con énfasis en la subsistencia de las sociedades indígenas y mestizas; la coexistencia de implantes productivos de carácter extensivo y comercial; la distribución de productos de consumo final e intermedio para los núcleos poblacionales urbanizados como enclaves administrativos que se sustentan por las inversiones y el funcionamiento del Estado (Gutiérrez et al., 2004). El perfil económico del departamento de Amazonas publicado por el DANE (2010) muestra que la actividad más sobresaliente es el comercio, seguido por los prestadores de servicios y la industria.

El departamento de Amazonas tiene una población total de 62.726 habitantes, 42.967 habitan en la zona rural y 25.659 en la zona urbana, cuya población está concentrada en Leticia. En cuanto a la composición étnica de la población, el 40,47\% está integrada por indígenas, mientras que el 50,85\% son no indígenas que proceden o son descendientes de población llegada de diferentes zonas del país (DANE, 2010).

\section{A PROPÓSITO DEL VALOR ECO- NÓMICO DE LA BIODIVERSIDAD}

En los ecosistemas naturales se encuentra una enorme riqueza para producir nuevos fármacos y alimentos (Figura 1). La oferta natural de algunos de estos productos se puede encontrar en los territorios que corresponden a resguardos indígenas. En este sentido, las comunidades deben hacer parte de las cadenas de valor que se puedan establecer, generando un beneficio económico para ellas. Se trata de Distribución Justa y Equitativa de Beneficios, tal como lo

\footnotetext{
${ }^{1}$ Bienes comunes se definen como aquellos recursos naturales o humanos de los cuales es difícil excluir a alguien o limitar su uso.
} 


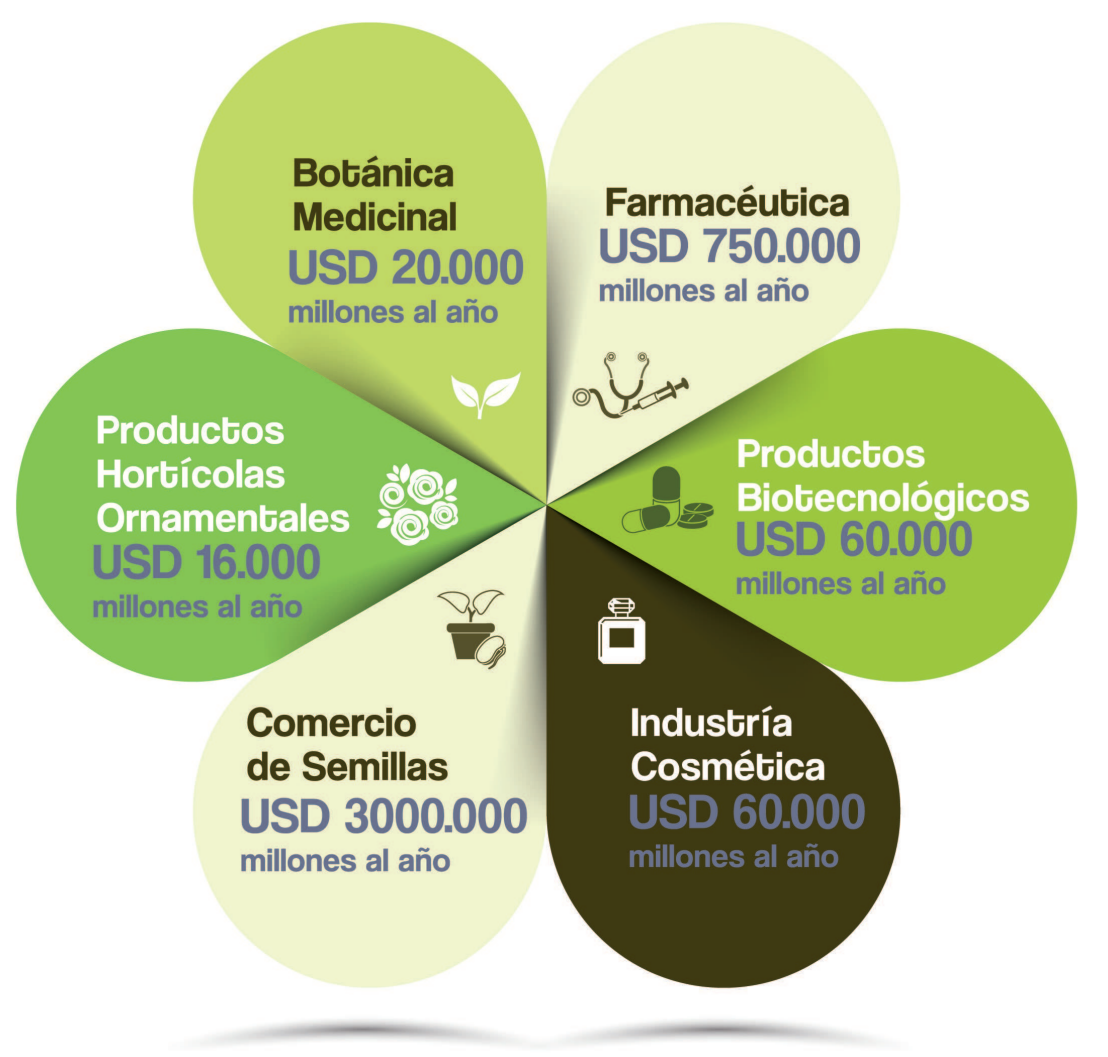

Figura 1. Valoración económica de la biodiversidad. Fuente: Elaboración propia a partir de (Kerry et al 1999).

considera la Convención de Diversidad Biológica, CDB.

De lo anterior, se debe inferir que la forma de implementar una política pública implicará entender cómo están representadas las poblaciones en el territorio, para que esta tenga resultados y se pueda cumplir con los objetivos que se proponen. Ya que son bienes comunes, una forma de hacerlo es construir con quienes están en el territorio. En la Política Ambiental diseñada para los Planes Nacionales de Desarrollo de tres gobiernos entre 2002 y 2014 se encuentra claramente identificado el PENMV, se incluye también la promoción de la biodiversidad y se promueve la articulación interinstitucional, así como la necesidad de la articulación para diferentes escenarios que se requieren para llevar adelante el PENMV. Igualmente, se habla de la importancia de la innovación incluyendo las formas de organización social y de las cadenas de valor.

Aunque es evidente la presencia del PENMV dentro del contexto de los planes nacionales de desarrollo sobre los cuales se direcciona la política nacional ambiental, estos no han sido la base para la realización de los planes de desarrollo departamental.

\section{Acerca de la articulación NACIONAL Y EL DEPARTAMENTO de Amazonas}

Al realizar una revisión de los Planes de Desarrollo del Departamento de
Amazonas entre los años 2002 al 2011, se encontró que en los periodos entre el 2002 y 2011, no se hace mención específica al PENMV, ni tampoco al modelo de gestión, en tanto que en el periodo extraordinario entre 2010 a 2011 "Un Amazonas con oportunidades para todos” aparece la referencia al PENMV, pero no se menciona un modelo de gestión y, en la articulación interinstitucional no hay referencia a las instituciones responsables frente al uso sostenible de la biodiversidad.

A partir de este análisis, se puede decir que los planes de desarrollo del departamento de Amazonas, entre el 2002 y el 2011, coinciden en que todos presentan una necesidad de utilizar la biodiversidad en el componente productivo, bajo el marco del desarrollo sostenible. Todos tienen en su visión el reconocimiento de la biodiversidad como factor de jalonamiento de riqueza y de mejoramiento de las condiciones socioeconómicas, dejando un horizonte de tiempo hasta el 2020. También reconocen en el medio ambiente una oportunidad para superar problemas de pobreza, pero solo lo enmarcan en el sector agropecuario, no en el tema ambiental, sin embargo, se generaron algunas experiencias orientadas al manejo sostenible de la biodiversidad con grupos locales, como se muestra más adelante.

\section{EXPERIENCIAS DESARROLLADAS CON COMUNIDADES LOCALES}

En este apartado se relacionan algunas experiencias de productos derivados del manejo sostenible de la biodiversidad, escogidos por sus características funcionales ${ }^{2} \mathrm{y}$, por ser emblemáticos de la biodiversidad del departamento. La Figura 2 ofrece información acerca de la forma en que funcionan los niveles de base de las comunidades y se da a conocer el esfuerzo que se debe hacer para trabajar con la biodiversidad

${ }^{2}$ Característica funcional: son alimentos con efecto potencial sobre la salud, además de su valor nutricional contienen nutrientes u otras sustancias que previenen o reducen el riesgo de enfermedades o disfunciones fisiológicas (Landstrom et al., 2009). 


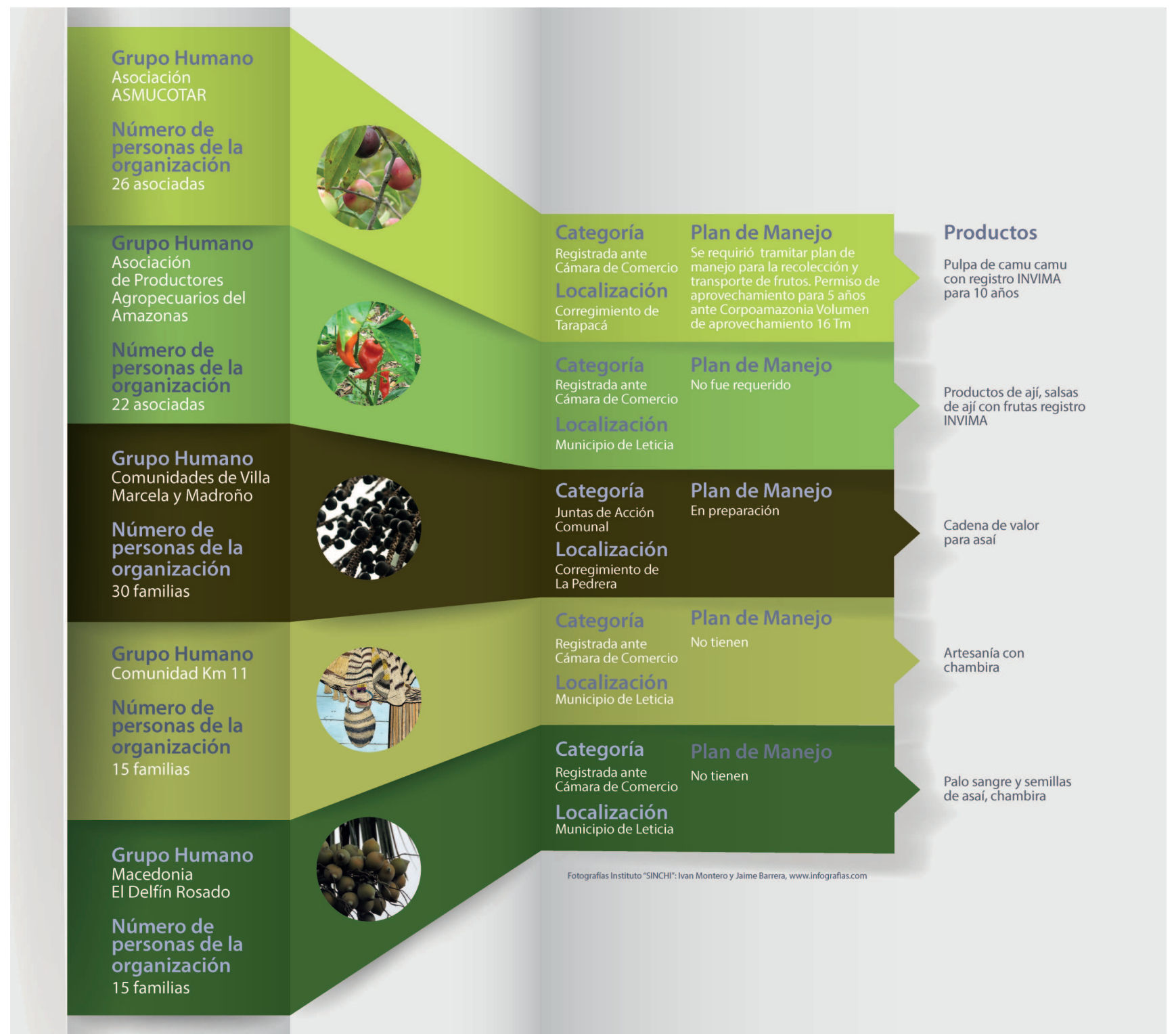

Figura 2. Experiencias desarrolladas con comunidades locales.

Fuente: Elaboración propia, 2015.

de la Amazonia. En el caso del camu camu, la base de información científica y de transferencia de tecnología que se debe generar para que el producto que se propone pueda ser ofertado en los mercados, se realicen los planes de manejo y se registre el desarrollo ante el INVIMA es alta y costosa.

OPINIÓN CALIFICADA DE LOS ACTORES INVOLUCRADOS EN EL DISEÑO DEL PENMV

Se incluye una síntesis de las entrevistas realizadas a la Doctora Claudia
Martínez Zuleta, viceministra de Ambiente en el período 1998-2002 y al Doctor John Alexander Bejarano Ortega, experto en temas de Biocomercio, quienes tuvieron relación directa con el PENMV. Estas entrevistas son importantes pues clarifican lo analizado en los planes departamentales de desarrollo 2002-2011 y hacen referencia a experiencias regionales desarrolladas en el departamento de Amazonas a partir del PENMV.

\section{LA VOZ DE LOS PROTAGONISTAS}

\section{Claudia Martínez Zuleta}

La doctora Martínez considera que el PENMV es una oportunidad para aprovechar la Biodiversidad en Colombia e impulsar los mercados verdes como instrumento de desarrollo desde la investigación y las políticas públicas. Este plan fue incluido en el proyecto colectivo ambiental, articulado al Plan Nacional de Desarrollo Cambio para 
construir la Paz 1998-2002 con un horizonte de tiempo de 10 años. Empezó en el 2002, nadie lo reconoció hasta después del 2006, ya que la oficina de mercados verdes desapareció del Ministerio de Ambiente y hubo cambio de política hacia la producción y el consumo sostenible donde se incluyó a mercados verdes pero quedó desarticulado. El modelo de gestión se concibió como una iniciativa del Orden Nacional, no se pensó en ella para lo Regional en la Amazonia pues había debilidades identificadas en el departamento de Amazonas.

La doctora Martínez opina que hay que pensar en grande, pues estas acciones necesitan mucho impulso y estrategia del Gobierno, además del sector privado. Respecto al Departamento de Amazonas, considera que hay muchas oportunidades pero también limitaciones dadas por la logística de la zona (transporte). Por lo anterior, recomienda definir lo estratégico como la Denominación de origen o hacer empresas tipo B Corporation que den estabilidad y sustentabilidad. Estas empresas buscan efectos positivos que ayuden al planeta. Es un reto pero es posible que tanto indígenas como colono-campesinos tengan más ingresos.

Como lecciones aprendidas, manifiesta que hubo asuntos que funcionaron, las Corporaciones hicieron actividades relativas a los Mercados Verdes igual que el Instituto SINCHI. La apuesta de la región es a ser competitivos y a aprovechar temas como geoturismo (Ecoturismo), reciclaje, priorizar líneas desde el orden nacional, tal como lo decidió Costa Rica con el lema de: "En Estado Puro” y la estrategia implementada por Perú que tiene hoy una fuerte competitividad en la producción de orgánicos.

\section{John Alexander Bejarano OrTEga}

El doctor Bejarano es contundente al afirmar que el PENMV es una oportunidad para la biodiversidad y el manejo sostenible del país. Mercados Verdes fue la política que contenía un amplio espectro de estrategias cuyo horizonte de tiempo era de 10 años. El Instituto Humboldt, por ejemplo, abre la línea de Biocomercio Sostenible desde el Biotrade de la United Nations Conference on Trade and Development, (UNCTAD), relacionado con la Convención de Diversidad Biológica (CDB), para evitar confusiones a la hora de articular estas líneas de investigación con las corporaciones autónomas regionales (CAR) y ellas lo incluyen en sus planes de acción trianual como: Mercados Verdes y Biocomercio.

El modelo de gestión no está articulado al departamento de Amazonas. Este tema, responsabilidad del Sistema Nacional Ambiental (SINA), aún está pendiente a pesar de ser un tema estructural. En el departamento de Amazonas es un reto lograr la articulación y el interés de las instituciones; les falta ser más concurrentes, más subsidiarias, más complementarias, más articuladas y a partir de allí generar modelos de uso en el territorio.

Las lecciones aprendidas a juicio del doctor Bejarano fueron entre otras:

- Más visión de mercado para los programas relacionados con la Biodiversidad.

- No temer tener ese enfoque, no puede ser solamente desde lo ambiental, debe incluir otros sectores que tengan en cuenta los principios de la conservación, pero que estén trabajando sobre el desarrollo.

- Mejorar la coordinación interinstitucional e intersectorial.

- El uso y desarrollo de los Bionegocios podrían convertirse en una ley de la Nación, sobre la base de la evolución de las políticas públicas.

- Mayor empoderamiento desde la investigación asociada al desarrollo, más visión de país desde la competitividad.

- Deben ser negocios donde la investigación tenga un gran nivel, como cambio climático, mejorar los presupuestos para este aspecto.
- No hay que poner en duda los servicios ecosistémicos de la selva amazónica, se pueden trabajar modelos endógenos, el departamento de Amazonas tiene potencial para mantener el patrimonio del país.

Las respuestas de los entrevistados expresan la necesidad de usar la biodiversidad en Colombia mediante el impulso a los mercados verdes. Además, coinciden en que el modelo de gestión del PENMV no estaba articulado al departamento de Amazonas. Por otra parte, están de acuerdo en que lo relacionado con los mercados verdes no pude ser realizado solo por lo ambiental y expresan que debe haber un fuerte liderazgo del gobierno nacional.

\section{Discusión}

El diseño de política que se definió para la implementación en el PENMV no tuvo en cuenta que tanto las comunidades como los pequeños productores también tienen desafíos para la implementación. El enfoque de arriba hacia abajo o Top Down implica imposición de valores externos a los pobladores locales y la aplicación de leyes generales y regulaciones generales a situaciones muy específicas (Sabogal et al., 2008).

Las arquitecturas institucionales se deben acondicionar para hacer la gobernanza más robusta; en este medio, las innovaciones y las estrategias adoptadas han ido evolucionando para construir acuerdos y confianza entre las instituciones y los pobladores locales. En lo relativo a la demanda, esta queda excluida cuando no considera los valores y condiciones de vida de dichas poblaciones. Hoy en día existen nichos de mercado para productos derivados de la biodiversidad provenientes de comunidades organizadas, con buenas prácticas de manufactura y con innovaciones en ciencia y tecnología.

El enfoque Top Down con el cual se diseñó el plan no permite la implementación de mercados verdes cuando excluye el "valor agregado" como atributo principal de las transacciones que los 
caracterizan. Dicho valor, al provenir de un proceso transformativo dependiente de reglas de uso común para el manejo sostenible de la biodiversidad, no puede ser impuesto por reglas generales y exógenas que lo desconocen.

El modelo de gestión necesario debe tener la capacidad de articular en un sistema de precios tradicional el "valor agregado” propio de los mercados verdes. Para lograr dicha articulación es necesario generar una "gobernanza adaptativa”, la cual requiere de instrumentos cuyo diseño incorpore, de manera horizontal, reglas adaptables que permitan arreglos institucionales flexibles a los intereses y participación de los diferentes actores.

Es de suma importancia entender que la población del departamento del Amazonas en su gran mayoría es indígena y que estos están organizados en 23 resguardos como propiedades colectivas, los cuales son inembargables, imprescriptibles e inalienables (Constitución Política de Colombia [Const]. Art. 63. Julio 7 de 1991. Colombia). Esto sugiere que la biodiversidad está en sus territorios, estas comunidades están organizadas y representadas por gobiernos propios e interculturales (Caciques y Gobernadores, Capitanes y/o Curacas), para el primer caso, y por Asociaciones de Autoridades Tradicionales Indígenas (AATIS), para el segundo caso.

Bajo las anteriores consideraciones es posible afirmar que la estrategia para la implementación del PENMV con el enfoque de Top down, con una entidad como el Fondo Biocomercio (Martínez, 2013), no está diseñada teniendo en cuenta las condiciones de inclusión requeridas para integrar a las comunidades indígenas y no se ve reflejado el postulado de partir de lo local. Por esta razón, es necesario entender que este Plan debió prever una mejor forma de articulación con el departamento de Amazonas, utilizando un enfoque de bienes comunes y dejando claro el papel tanto de las instituciones, como de las comunidades. En el mismo sentido, el PENMV no consideró los beneficios de la cooperación para su implementación.

Las metas formuladas por el PENMV cumplen parcialmente con lo correspondiente al fortalecimiento y a la ampliación de la oferta de productos verdes y su comercialización, pues, las restantes metas, como la adecuación de la oferta existente hacia productos más verdes y el fortalecimiento de la participación de productos más verdes en las exportaciones colombianas no tradicionales, están lejos de cumplirse por la falta de articulación con otros sectores, tanto nacionales como departamentales.

Los planes de desarrollo con excepción del correspondiente al 2010-2011, "Un Amazonas con oportunidades para todos" no tienen una mención expresa al PENMV. Esto indica que no se hizo una gestión adecuada por quienes propusieron en su momento este Plan. Desde la perspectiva real, no se articuló el PENMV de manera apropiada dentro de la región; sin embargo, la agenda de competitividad sí reemplazó este papel y esa es la razón por la cual en los planes donde este elemento empezó a identificarse, se produjo una entrada de forma explícita a la acción de darle forma a la biodiversidad. Entre tanto, la canasta de productos amazónicos que se ve reflejada en esta Agenda da forma al proceso, que, como bien lo dice el Plan de Desarrollo "Entre todos podemos”, contó con la participación de un grupo de instituciones del orden regional, luego de haber recibido la visita al departamento de Amazonas del doctor Fabio Valencia Cossio, quien era el presidente de la Agenda Nacional de Competitividad. $^{3}$

Los entrevistados coinciden en que debe haber un liderazgo del orden nacional y un expreso llamado a que esta sea una ruta que deben emprender los gobiernos nacionales para la Amazonia pues, aunque en los Planes Nacionales de Desarrollo existe una mención expresa sobre el manejo sostenible de la biodiversidad, los Mercados Verdes y el Biocomercio, el nivel departamental no lo incorporó.

Queda claro que la forma como debe hacerse la articulación entre lo público y lo privado no se da en la escala regional. Pese a que existen un sinnúmero de comités, no existe uno que se dedique estrictamente a discutir las perspectivas de desarrollo sostenible. Los comerciantes que están en la región no ven la utilidad de promover empresas basadas en la biodiversidad y que apunten a una forma de emprendimiento diferente.

De igual manera, las respuestas de los entrevistados contribuyen a comprobar la falta de articulación interinstitucional y a identificar la carencia del modelo de gestión. Igualmente, muestran la percepción de debilidad de los gobiernos departamentales, los efectos que tuvieron los cambios de política nacional pues, se desarticuló el PENMV de la propia política nacional de producción y consumo sostenible, y la insistencia por parte de los entrevistados en que esta es una responsabilidad del gobierno nacional, sin perder de vista la oportunidad que para el Amazonas tiene la biodiversidad.

La Figura 2 presenta las evidencias de las experiencias regionales derivadas del PENMV que han tenido impacto local pero, no se registra como logro en los planes de desarrollo departamentales pues, los planes analizados carecen de metas. Para las instituciones del orden nacional como Corpoamazonia y el Instituto SINCHI que son parte del Sistema Nacional Ambiental, SINA, las metas están inscritas en sus Planes Trianuales de gestión y en el Plan Estratégico respectivamente. En este último caso, con el apoyo del presupuesto general de la nación y la cooperación internacional. Lo anterior permite medir

\footnotetext{
${ }^{3}$ Situación confirmada en la entrevista telefónica con el Doctor John Bejarano Ortega.
} 
el alcance de sus acciones y mostrar logros efectivos en la región.

Todas estas experiencias han requerido acciones en el largo plazo, así como espacios de concertación con los pobladores locales para su permanencia en el tiempo y su trascendencia en el bienestar de los grupos humanos organizados. El esfuerzo institucional por parte del Instituto SINCHI y Corpoamazonia ha sido fundamental, de largo plazo y con gestiones recurrentes, subvaloradas y no suficientemente reconocidas por los gobiernos departamentales, aun cuando han estado inscritas en los bancos de proyectos del Departamento Nacional de Planeación en congruencia con los planes nacionales de desarrollo. El balance de todas estas acciones indica que a pesar de las limitaciones es posible el desarrollo humano y organizacional a partir del uso sostenible de la biodiversidad como una alternativa para el desarrollo productivo de la región.

Otro aspecto importante para resaltar es que el concepto de desarrollo es diferente tanto para lo nacional, como para lo regional y lo local; las comunidades lo expresan de una forma que no corresponde a los intereses de cumplir con los objetivos de políticas, en este caso del PENMV, lo cual no implica que estas iniciativas sean imposibles de lograr. Se demuestra también que las actividades se cumplen ya que, están incluidas en el Plan Estratégico de las instituciones vinculadas al SINA: Instituto SINCHI y Corpoamazonia.

Estas instituciones conocen el PEN$\mathrm{MV}$, además saben que la relación con las organizaciones requiere un alto grado de articulación y negociación.

\section{ConClusión}

El PENMV no fue implementado de manera adecuada en el departamento de Amazonas pues, no se incluyó un modelo de gestión en los planes de desarrollo de los gobiernos departamentales. El Gobierno departamental no tuvo en cuenta el PENMV para orientar decisiones de política regional sobre el aprovechamiento sostenible de la biodiversidad, por lo tanto no existió articulación institucional para la implementación del PENMV, ya que el diseño institucional restringe la participación y la articulación que se requiere desarrollar para generar estrategias de concertación con los gobiernos indígenas. Lo anterior permite reconocer las diferencias y entender sus propios sistemas de gobernanza y relación con los recursos naturales que están en sus resguardos indígenas.

Aunque los planes de desarrollo departamental no incluyeron el PENMV dentro de su planeación, las instituciones del orden nacional que tienen representación en la Amazonia sí lo hicieron. Ellas desarrollaron estrategias de articulación que permitieron demostrar que sí es posible el manejo sostenible de la biodiversidad, en alianza con las comunidades locales, para la configuración de mercados verdes. Para ello se introdujo el concepto de gobernanza adaptativa, que permite concertar las necesidades de información, capacitación y transferencia de tecnología, entre otras, con dichas comunidades. Esto permitió obtener productos derivados de la biodiversidad, para los cuales se requería la articulación directa entre el departamento y la nación, y de esta forma lograr la participación intersectorial necesaria para su implementación.

La participación intersectorial es necesaria porque el uso sostenible de la biodiversidad no es solo responsabilidad de lo ambiental, se requiere la participación de varios sectores que promuevan y articulen intereses y recursos con el plan de desarrollo departamental de manera clara en una zona geográfica, que tiene unas ventajas importantes desde el horizonte de la biodiversidad.

De igual forma, las experiencias regionales que desarrollaron las entidades como el Instituto SINCHI y Corpoamazonia en el departamento de Amazonas dan cuenta de una construcción de arquitecturas interinstitucionales y de procesos de concertación con las comunidades, que son pilares fundamentales para garantizar el éxito de los emprendimientos. Estas experiencias deben ser incorporadas como ejemplos exitosos a los planes de desarrollo departamentales para que la biodiversidad sea considerada una posibilidad de desarrollo sostenible a partir de los mercados verdes.

\section{REFERENCIAS}

Aguilar, L. (1993). La implementación de las políticas. México: Porrúa.

Barriga, M., \& Campos, J. (2007). Gestión ambiental en América Latina. Experiencias y aprendizajes: gobernanza adaptativa y colaborativa en bosques modelo, cuencas hidrográficas y corredores biológicos. Turrialba: CATIE.

Bejarano, J. (26 de Febrero de 2006). Entrevista con el Director de la Agenda Nacional de Competitividad del Departamento del Amazonas. (L. M. Mantilla, Entrevistador)

Cárdenas, D., et al. (2006). Botánica en la Amazonia colombiana: Doscientos años después de Martius. Colombia amazónica: Edición especial , 71 - 100.

Departamento Administrativo Nacional de Estadísticas. (2010), Bogotá, Colombia.

Departamento Nacional de Planeación. (2002 - 2005). Plan de Desarrollo Departamental: Un amazonense por un Amazonas con futuro, Bogotá, Colombia.

Departamento Nacional de Planeación. (1998 - 2002). Plan Nacional de Desarrollo: Cambio para Contruir la Paz, Bogotá, Colombia.

Departamento Nacional de Planeación. (2006 - 2012). Plan Nacional de Desarrollo: Estado comunitario: desarrollo para todos, Bogotá, Colombia.

Departamento Nacional de Planeación. (2002 - 2006). Plan Nacional de Desarrollo: Hacia un Estado Comunitario, Bogotá, Colombia. 
Dini, M. (2010). Competitividad: Redes de empresas y cooperativa empresarial (Serie Gestión Pública No. 72 ed.). CEPAL.

Ferraro C, C. (2010). Políticas de articulación productiva en América. Chile: CEPAL - FUNDES.

Gutiérrez R, F., Acosta M, L. E., \& Salazar C, C. A. (2004). Perfiles urbanos en la Amazonia colombiana. Bogotá: Instituto Sinchi.

Instituto Amazónico de Investigaciones Científicas. (21 de Febrero de 2013). Base de Datos Inírida: Población total del departamento de Amazonas proyectada a 2010. Disponible en; http://sinchi.org.co/inirida/sinchi/ consultas/calculo_lista1.php.
Instituto Amazónico de Investigaciones Científicas. (2013). Mapa de resguardos indígenas y asentamientos Amazonia colombiana.

Martinez, C. (26 de Febrero de 2013). Entrevista a la Viceministra de Medio Ambiente. (L. M. Mantilla, Entrevistador)

Ministerio del Medio Ambiente. (2002). Plan Estratégico Nacional de Mercados Verdes. Bogotá, Colombia.

Ostrom, E. (2002). El gobierno de los bienes comunes: La evolución de las instituciones de acción colectiva. Región y Sociedad, XIV (24).

Parsons, W. (2007). Políticas públicas: Una introducción a la teoría y la práctica del análisis de políticas públicas. México: Flacso.
Pressman, J., \& Wildasvsky, A. Implemetation (3 ed.). Berkeley: Press Eds.

Sabogal, C., De Jong, W., Pokorny, B., \& Louman, B. (2008). Manejo forestal comunitario en América: Ecperiencias, lecciones y retos para el futuro. Belém, Brasil: CIFOR - CATIE.

Stein, E. (2006). La política de la políticas públicas. Washington: Banco Interamericano de Desarrollo.

Strein, E., \& Tomassi, M. (2006). La política de las políticas públicas. Política y Gobierno, XIII (2), 396 - 416.

World Resources Institute (2005). Ecosystems Millennium Assessment: Ecosystems and Human Well-being. Washington: WRI. 